\title{
Plasmonically Coupled Faraday Effect in Fe- and Au-doped Silicate Glasses Irradiated with Femtosecond Laser
}

\author{
Seisuke NAKASHIMA ${ }^{* 1,{ }^{*}}$, Koji SUGIOKA ${ }^{* 1}$, Katsumi MIDORIKAWA ${ }^{* 1}$, and Kohki MUKAI ${ }^{* 2}$ \\ ${ }^{* 1}$ Advanced Science Institute, RIKEN, 2-1 Hirosawa, Wako, Saitama 351-0198, Japan \\ E-mail:nakashima@ynu.ac.jp \\ ${ }^{* 2}$ Yokohama National University, 79-5 Tokiwadai, Hodogaya-ku, Yokohama-shi, Kanagawa 240- \\ 8501, Japan
}

\begin{abstract}
The optical properties and the magneto-optical responses were space-selectively modified in transparent $\mathrm{Fe}^{3+}$ - and $\mathrm{Au}^{3+}$-doped glasses by irradiation with an infrared femtosecond laser and twostep annealing. The primary annealing at $450{ }^{\circ} \mathrm{C}$ induced the precipitation of magnetic spinel-typed $\mathrm{Fe}$-oxide nanoparticles, and the precipitation of Au nanoparticles occurred during the secondary annealing at $550{ }^{\circ} \mathrm{C}$. As a result, the irradiated and annealed glasses shows localized surface plasmon resonance absorption at around $510 \mathrm{~nm}$, and also plasmonically coupled peaks at almost the same wavelength in Faraday rotation spectra. As increasing the pulse energy, the position of the localized surface plasmon resonance (LSPR) peak slightly shifted into the shorter wavelength, which is due to the decrease in the size of Au nanoparticles. The LSPR-coupled Faraday peaks were observed as a negatively enhanced rotation, which suggests that ferrimagnetic contribution to the magneto-optical properties was enhanced by the electric field on the surface of Au nanoparticles. This coupling phenomenon emerged as a result of a modification of composition of host glasses.
\end{abstract}

DOI: $10.2961 /$ jlmn.2014.02.0010

Keywords: Femtosecond laser, Ferrimagnetic nanoparticle, Superparamagnet, Plasmonic material, Localized surface plasmon resonance

\section{Introduction}

Magnetoplasma surface wave have recently attracted much interest as an intriguing research field due to the direct coupling between the plasomon and magnetooptical effect. Enhancement of magneto-optical responses by the localized surface plasmon resonance (LSPR) can provide extensive applications in efficient optical isolaters, ultrahigh-density recording devices, and spatial light modulators, and so on [1]. In many cases, the plasmonic enhancement of magnetooptical effect is observed in nanocomposite structures with plasmonic metal and magnetic metal [2] or plasmonic metal and magnetic oxide [3] systems. Therefore, a variety of nanostructures, such as layer-by-layer thin films [4], nanoisland on the thin films [5], and core-shell nanoparticles [6], have been studied using physical deposition processes or chemical syntheses. Although they have been discussed the relationship between magnetooptical effect and enhanced electric field on the metal nanostructure due to plasmon polariton at the phase boundary, the detailed mechanism is still open.

Femtosecond (Fs) lasers have been widely utilized as powerful tools for sensing optical properties or a spatially selective modification of various physical properties of materials, including optical, electrical, mechanical, and magnetic properties. In particular, focused fs lasers inside transparent glasses induce a variety of interactions with atoms and ions, such as photo-reduction, photo-excitation, heat accumulation, atomic diffusion and so on [7-10]. In recent years, we have reported that the silicate glasses codoped with Fe-oxide and $\mathrm{Al}$ irradiated with a fs laser and subsequent annealing exhibit an increase in the saturation magnetization [11], localized surface plasmon resonance (LSPR) in absorption spectra, and plasmonically enhanced magnetooptical Faraday response [12]. It can be explained in terms of nanocompsite structures of ferrimagnetic magnetite and Al nanoparticles. Negative peaks at around resonance wavelength in the Faraday rotation spectra strongly suggest that direct coupling between plasmonic and ferrimangetic contributions. In contrast to this, fs-laser irradiation process to $\mathrm{Fe}$-oxide- and $\mathrm{Au}^{3+}$ ions-codoped glasses caused a positively enhanced diamagnetic Faraday rotation. To improve this, a two-step annealing process was proposed, and the effectiveness was confirmed [13]. In this paper, we have investigated an optimum composition of the host glass to observe a direct coupling between ferrimagnetic Faraday effect and LSPR by fabricating a nanocomposites of $\mathrm{Fe}$-oxide and $\mathrm{Au}$ nanoparticels using a fs-laser irradiation process.

\section{Experimental}

Soda lime silicate glass doped with $\mathrm{Fe}^{3+}$ (hematite) and $\mathrm{Au}^{3+}$ was prepared by melt-quenching method. The glass has a composition of (100-3x) $\mathrm{SiO}_{2} \cdot 2 x \mathrm{Na}_{2} \mathrm{O} \cdot x \mathrm{CaO}(\mathrm{mol} / \%)$, which is doped with $0.05 \alpha-\mathrm{Fe}_{2} \mathrm{O}_{3}$ and $0.02 \mathrm{HAuCl}_{4}$. Reagent-grade $\mathrm{SiO}_{2}, \mathrm{Na}_{2} \mathrm{CO}_{3}, \mathrm{CaCO}_{3}, \alpha-\mathrm{Fe}_{2} \mathrm{O}_{3}$, and $\mathrm{HAuCl}_{4}$ were used as starting materials. A mixture of these powders was melted in a platinum crucible at $1550{ }^{\circ} \mathrm{C}$ for $1 \mathrm{~h}$ in air. The melt was then quenched into a glass by casting it onto a stainless-steel plate at room temperature. The glass samples with the thickness of about $3 \mathrm{~mm}$ were prepared, both sides of which were polished so that optical responses can be measured at the transmission configuration. 


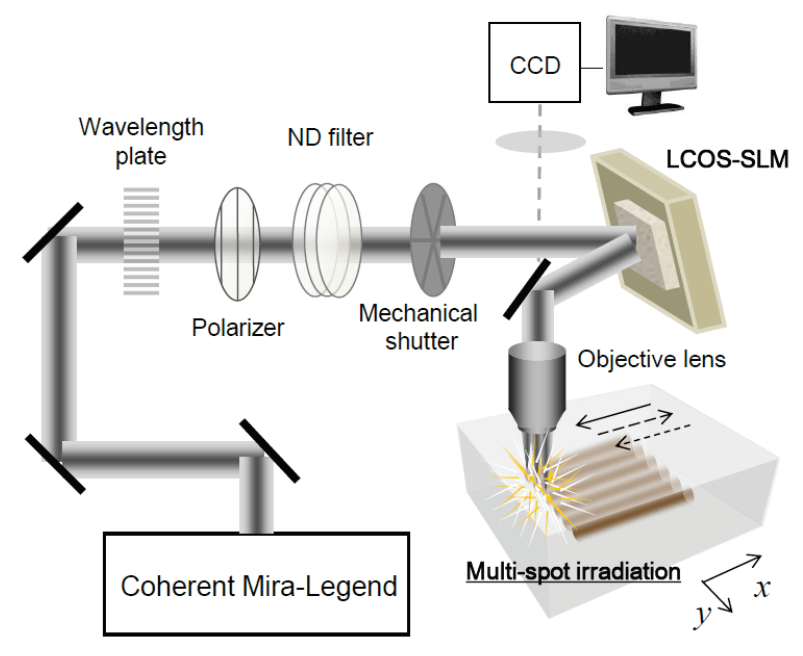

Fig. 1 Schematic illustration of laser-irradiation processes.

Femtosecond-laser irradiation was carried out to form the nanocomposites of $\mathrm{Fe}$-oxide and metallic $\mathrm{Au}$ nanoparticles inside the glass materials (Fig. 1). Near-infrared fslaser pulses (Mira-Legend, Coherent Inc.; wavelength: 800 $\mathrm{nm}$; pulse widths: 120 fs (full width at half maximum); repetition rate: $1 \mathrm{kHz}$ ) were focused inside the glass samples with an objective lens $(f=10 \mathrm{~mm}, \mathrm{NA}=0.45$; LUPlan, Nikon). The focused position is $1 \mathrm{~mm}$ below the upper surface of the sample, and the spot size of focal point is about $2.68 \mu \mathrm{m}$. The glass samples on a PC-controlled $X Y$ translation stage were horizontally translated in the $X$ direction at a scan speed of $1000 \mu \mathrm{m} / \mathrm{s}$. Laser-irradiated lines with pitches of $5 \mu \mathrm{m}$ in the $Y$ direction were produced, resulting in a square irradiated area with about 4-mm-long sides. To enhance the processing efficiency, we have utilized a liquid crystal on silicon-spatial light modulator (LCOS-SLM, X10468-02, Hamamatsu Photonics K.K.) system. When a fs-laser beam is reflected on the SLM, the spatial phase distribution of the laser beam is modulated. After this modulation, the laser beam passes through a telescope (not shown), which consisted of two concave lenses (magnification of $\mathrm{M}=0.3$ ). Then, focusing with the objective lens creates multiple focused beam spots. In this experiment, 5 spots with pitches of $20 \mu \mathrm{m}$ were created and repeatedly scanned every $5 \mu \mathrm{m}$. After 4-time scanning, 100$\mu \mathrm{m}$ wide area is filled with 20 lines every $5 \mu \mathrm{m}$. Therefore, the processing efficiency was increased fivefold. After the laser irradiation, the glass samples were annealed in a twostep annealing process consisting of 90 -minute-anneal at $450{ }^{\circ} \mathrm{C}$ and subsequent 30 -minute-anneal at $550{ }^{\circ} \mathrm{C}$ for the purpose of efficient and individualized precipitation of ferrimangetic magnetite and $\mathrm{Au}$ nanoparticles[13]. The absorption spectra of glass samples were measured using a spectrophotometer (JASCO V-570). The wavelength was changed from 300 to $900 \mathrm{~nm}$. Faraday rotation angles as a function of wavelength were measured using a commercially available measurement system for Faraday and Kerr effects (JASCO, Model K-250).

\section{Results and discussions}

By changing the chemical composition of the host silicate glass from basic composition $(x=10)$, the ionicdiffusion behavior can be modified, realizing different pre-
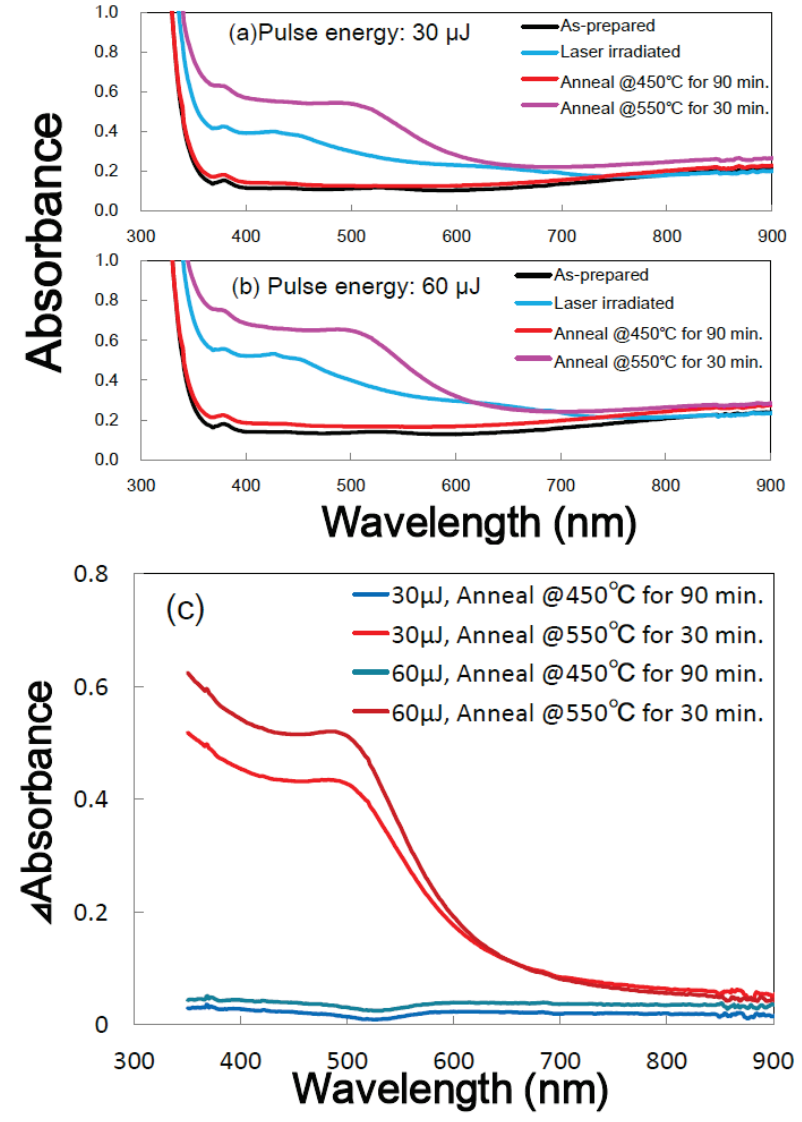

Fig. 2 Optical absorption spectra for as-prepared, irradiate, and two-step-annealed glass samples in the pulse energies of (a) $30 \mu \mathrm{J}$ and (b) $60 \mu \mathrm{J}$. (c) Difference absorption spectra between the as-prepared and two-stepannealed samples.

cipitation conditions of nanoparticles. With the increase in the fraction of alkali oxides $(x \sim 14)$, the obtained glass became muddy brown. Due to this opaqueness, alkali-rich compositions are not suitable for the laser irradiation process. On the other hand, the decrease in the composition of alkali oxide $(x \sim 6)$ induced red colorization after quenching. This is ascribed to the precipitation of $\mathrm{Au}$ nanoparticles. It is presumed that the melt was inhomogeneously mixed due to the high viscosity and $\mathrm{Au}$ atoms reduced in hightemperature furnace could not fully react with oxygen, resulting $\mathrm{Au}$ nanoparticles. In the meanwhile, transparent glasses were obtained in the parameter, $x \leq 8.5$. Therefore, we used the glass $(x=8.5)$ for the laser irradiation.

Figure 2 shows the absorption spectra in the pulse energy of (a) $30 \mu \mathrm{J}$ and (b) $60 \mu \mathrm{J}$, measured for the asprepared samples, laser-irradiated, annealed at $450^{\circ} \mathrm{C}$ for 90 min, and additionally annealed at $550^{\circ} \mathrm{C}$ for $30 \mathrm{~min}$, respectively. The difference absorption spectra between the asprepared and annealed samples are also shown in Fig. 2 (c). The as-prepared glass doped with $\mathrm{Fe}^{3+}$ and $\mathrm{Au}^{3+}$ exhibits good optical transparency. The absorption spectrum (black curve) has no absorption at visible wavelengths longer than $350 \mathrm{~nm}$. This transparency of the glass is realized as aresult of the small absorption cross section of trivalent $\mathrm{Fe}^{3+}$ ions, which is due to all $d-d$ transitions being spin forbidden in a high-spin configuration. Prior to the laser irradiation, the effects of anneal process (without irradiation) were investi- 


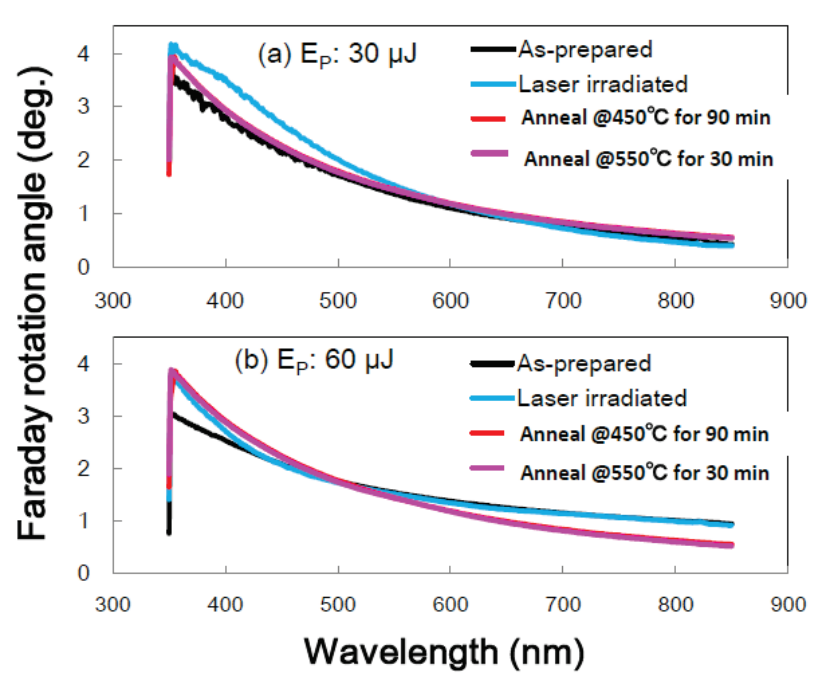

Fig. 3 Faraday rotation angles as a function of wavelength for as-prepared, fs-laser-irradiated and two-step-annealed glass samples in the pulse energies of (a) $30 \mu \mathrm{J}$ and (b) $60 \mu \mathrm{J}$.

gated. The color of the glass was totally changed into deep red after the annealing at the temperature higher than 550 ${ }^{\circ} \mathrm{C}$ for $30 \mathrm{~min}$, which is due to the precipitation of $\mathrm{Au}$ nanoparticles. Meanwhile, the color change was not observed by the annealing below $500{ }^{\circ} \mathrm{C}$ for $30 \mathrm{~min}$ (data were not shown).

When the glass sample was irradiated with fs-laser in both pulse energies, the focused region inside the glass was locally turned into brown. The spectrum for irradiated glass (blue curve in Fig. 2 (a) and (b)) shows broad absorption peaks at around 440 and $650 \mathrm{~nm}$, which are attributable to hole-trap centers (e.g., $\mathrm{HC}_{1}$ and $\mathrm{HC}_{2}$ ) at nonbridging oxygens. $\mathrm{HC}_{1}$ and $\mathrm{HC}_{2}$ are holes trapped at the nonbridging oxygen in the $\mathrm{SiO}_{4}$ polyhedron with two and three nonbridging oxygens, respectively $[14,15]$. At this time, free electrons were driven out from the $2 p$ orbital of the nonbridging oxygen via multiphoton absorption process within three-dimensionally confined space. By applying thermal stimulation, neighboring $\mathrm{Fe}^{3+}$ or $\mathrm{Au}^{3+}$ ions have chances to trap the free electrons and to be partially reduced to $\mathrm{Fe}^{2+}$ ions or Au atoms [16].

In our previous paper, we have suggested that the twostep annealing process after laser irradiation is effective in obtaining multi-phase nanoparticle composites such as magnetite and $\mathrm{Au}$. In fact, a single annealing at $550{ }^{\circ} \mathrm{C}$, which causes the precipitation of $\mathrm{Au}$ nanoparticles in the overall regions, allows worse spatial selectivity. As shown in red curves in Fig. 2(a) and 2(b), the absorption spectra after the primary annealing at $450{ }^{\circ} \mathrm{C}$ are almost similar to those for the as-prepared samples. The hole trap centers almost completely disappeared and transparency was recovered. It is presumed that the generated free electrons were trapped by the hole centers on oxygens and other metal ions such as $\mathrm{Fe}^{3+}$ and $\mathrm{Au}^{3+}$. It is also clear that $\mathrm{Au}$ nanoparticles are not precipitated under the lowtemperature annealing, although some of $\mathrm{Au}$ ions are reduced to $\mathrm{Au}$ atoms. In this step, ferrimagnetic Fe-oxide, such as magnetite, nanoparticles were preferentially precipitated, which is similar to the situation that Fe-oxide-doped

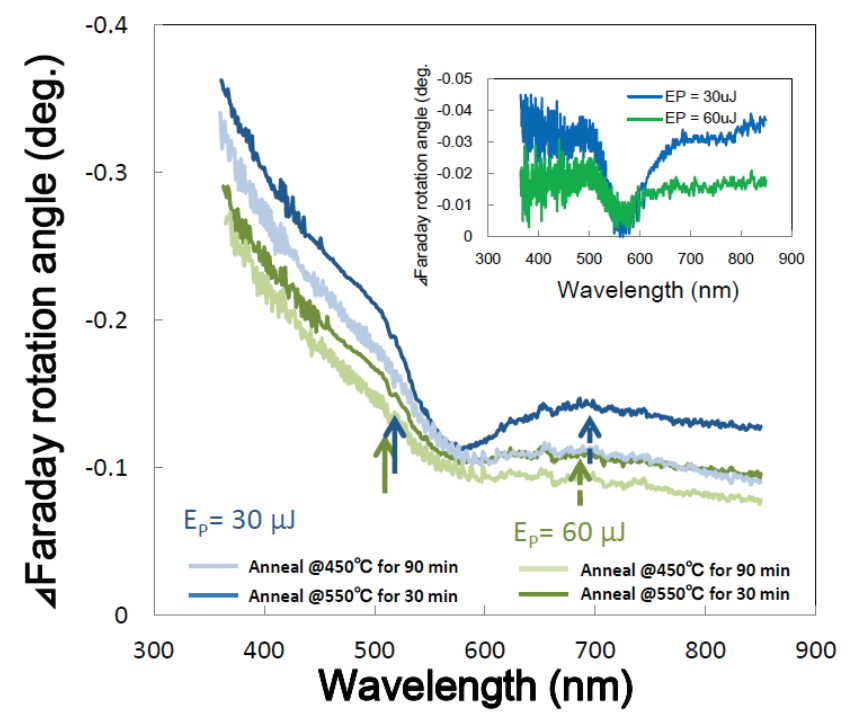

Fig. 4 Difference spectra in the Faraday rotation angle as a function of wavelength between the as-deposited and two-step-annealed samples. Inset plots the increment in Faraday rotation as a function of wavelength between high- and low-temperature annealing.

silicate glasses irradiated with fs laser provides a significant enhancement of saturation magnetization at room temperature in our previous report [11]. In the meanwhile, optical spectra for the samples after secondary annealing at $550{ }^{\circ} \mathrm{C}$ show distinct peaks at 511 and $509 \mathrm{~nm}$ for pulse energies of 30 and $60 \mu \mathrm{J}$, respectively. These peaks can be ascribed to LSPR absorption peaks due to precipitated $\mathrm{Au}$ nanoparticles. In this step, Au atoms reduced in the previous annealing step were aggregated into $\mathrm{Au}$ nanoparticles. As the increase in the pulse energy, the amount of crystalline nucleus increases, resulting in the decrease in the amount of $\mathrm{Au}$ atoms available for the grain growth. That is why the size of precipitated Au nanoparticles for the pulse energy of $60 \mu \mathrm{J}$ is smaller than that for $30 \mu \mathrm{J}$. The irradiated regions inside the glass are locally colored into faint red. Hence, the colorization in the unirradiated regions was suppressed even after the annealing at $550{ }^{\circ} \mathrm{C}$. Thus, crystallization of $\mathrm{Fe}$-oxide nanoparticles occurs during the annealing at $450{ }^{\circ} \mathrm{C}$ prior to precipitation of $\mathrm{Au}$ nanoparticles. Fig. 2(c) shows the difference spectra between the annealed and the as-prepared glass samples. It is clear that the primary annealing at low temperature gives no change in absorption spectra and the secondary annealing induces drastic precipitation of Au nanoparticles.

Magneto-optical Faraday effect can be enhanced by the existence of plasmonic $\mathrm{Au}$ nanoparticles as nanocomposites with magnetite nanoparticles precipitated in the glass matrix. To investigate the interaction between magnetic and plasmonic properties, magneto-optical Faraday rotation as a function of wavelength was measured under an external magnetic field of $1.5 \mathrm{~T}$ for each sample. Figure 3 shows the Faraday rotation spectra for the as-prepared, laser-irradiated and subsequent annealed samples in the pulse energies of (a) $30 \mu \mathrm{J}$ and (b) $60 \mu \mathrm{J}$. After irradiation, the Faraday spectra were clearly changed from those for the as-prepared glass, particularly in visible wavelength range. This enhancement of rotation angles is directly related with the increase in absorption coefficient based on hole 
trap centers, although the reason still remains unclear at present. The difference Faraday spectra between the asprepared and the annealed samples are shown in Fig. 4. In both pulse energies, the spectra for the samples after hightemperature annealing show broad negative peaks at around $511 \mathrm{~nm}\left(\mathrm{E}_{\mathrm{P}}=30 \mu \mathrm{J}\right)$ and $506 \mathrm{~nm}\left(\mathrm{E}_{\mathrm{P}}=60 \mu \mathrm{J}\right)$. This enhancement is attributed to the existence of LSPR based on $\mathrm{Au}$ nanoparticles, since the peak position is well consistent with that of LSPR absorption. In ferrimagnetic Feoxides with spinel structures, such as magnetite, the origin of magnetism is ordering of Fe ions. Although the $d-d$ transitions of $\mathrm{Fe}^{3+} 3 d$ electrons are basically both spin- and parity-forbidden, these transitions were slightly allowed due to ferrimagnetic ordering under an external magnetic field. In this case, there is a small contribution to the optical absorption and magneto-optical response in the wavelength range of 480-550 $\mathrm{nm}$. These weakly allowed transitions are enhanced by the LSPR absorption due to Au nanoparticles in the vicinity of magnetite, which leads to observation of broad peaks in Faraday rotation spectra. Compared with the spectra for the annealed at low temperature, the Faraday rotation is enhanced at around not only $500 \mathrm{~nm}$ but also $700 \mathrm{~nm}$ after secondary annealing at $550{ }^{\circ} \mathrm{C}$. This is emphasized by the inset of Fig. 4, which plots the increment in Faraday rotation between high and low temperature annealing as a function of wavelength. The enhancement in the longer-wavelength range does not correspond to the results of absorption spectra. Therefore, this is presumably due to the intrinsic enhancement of ferrimagnetic phase [17] rather than the plasmonically coupled modification of magnetooptical effect.

In our previous report, we observed peaks with positive values in the glasses with the composition of $x=10$, which can be explained in terms of major contribution due to plasmonically coupled diamagnetic Faraday effect. The reason why the coupling between $\mathrm{Au}$ and Fe-oxide nanoparticles is weak is presumably attributable to the long distance of these nanoparticles. When the laser beam is focused inside the glasses, photoreduction of $\mathrm{Au}$ and $\mathrm{Fe}$ ions and ionic diffusion occurs due to heat gradient. Generally, ionic species move toward the surrounding area from the central region of the focal point. The behavior of ionic diffusion in glasses is strongly related with the ionic radius and the bonding properties of the glass network. In the case of $\mathrm{Au}$ ions, the ion radius is significantly larger $\left(\mathrm{Au}^{3+}: 0.85\right.$ $\left.\AA, \mathrm{Au}^{1+}: 1.37 \AA\right)$ compared with the $\mathrm{Fe}^{2+}(0.63 \AA)$ and $\mathrm{Fe}^{3+}$ $(0.55 \AA)$ ions. Hence, these two species experience different diffusion length, resulting in long distance between the $\mathrm{Au}$ and $\mathrm{Fe}$-oxide nanoparticles. In contrast, radius of $\mathrm{Al}$ ion $(0.54 \AA)$ is similar to those of Fe ions. That is why we successfully produced the nanoparticle composites in Feoxide- and Al-doped silicate glasses. In this work, we have increased the concentration of $\mathrm{SiO}_{2}$ with the decrease in those of alkali oxides. The modification of the glass composition tends to lead to the increase in the number of Si-OSi bonds and to strengthen the 3-dimensional glass network due to the decrease in the alkali ions. In this case, the relatively strong glass network prevents ionic diffusion of Fe ions, compared with the glass containing weaker network due to larger amount of $\mathrm{Si}^{-} \mathrm{O}^{-}$. As a result, the distance between precipitated magnetite and $\mathrm{Au}$ becomes shorter, and the coupling between the ferrimagnetic contribution and the LSPR based on the plasmonic Au nanoparticles is established, leading to the negatively enhanced Faraday rotation.

\section{Conclusions}

Spatially selective modifications of the optical property and the magneto-optical Faraday response were performed by the fs-laser irradiation process in transparent $\mathrm{Fe}^{3+}$ - and $\mathrm{Au}^{3+}$-doped silicate glasses. The irradiation with fs laser and two-step-anneal procedures gave rise to the precipitation of Au nanoparticles showing LSPR at the wavelengths of about $510 \mathrm{~nm}$ in the absorption spectra. Faraday rotation angles as a function of wavelength were measured and the distinct negative peaks were observed at almost the equivalent wavelength for the two-step annealed samples, suggesting that the coupling between the LSPR due to the $\mathrm{Au}$ nanoparticles and the ferrimagnetic magnetite nanoparticels was realized. The primary annealing at $450{ }^{\circ} \mathrm{C}$ contributes to the precipitation of magnetite nanoparticles. Au nanoparticles were grown by the secondary annealing at $550{ }^{\circ} \mathrm{C}$. It is considered that the modification of the glass composition is effective in controlling the ionic diffusion. By increasing the silica component, 3-dimensional glass network became relatively strong. Therefore, the magnetite and $\mathrm{Au}$ nanoparticles were precipitated in spatially closer positions, and the coupling between the ferrimagnetic Faraday effect and the LSPR was detected as the negative Faraday rotation peaks.

\section{Acknowledgement}

The experiments using fs laser with LCOS-SLM were carried out with the help of Prof. M. Sakakura, Prof. Y. Shimotsuma, and Prof. K. Miura in Kyoto University. The measurements of Faraday effect were performed with the help of Prof. K. Tanaka in Kyoto University. This work was financially supported by a Grant-in-Aid for Young Scientist B (No. 24760544) from the Ministry of Education, Culture, Sports, Science, and Technology (MEXT), Japan, and partially supported by the Asahi Glass Foundation, and by the Futaba Electronics Memorial Foundation.

\section{References}

[1] M. Inoue, R. Fujikawa, A. Baryshev, A. Khanikaev, P. B. Lim, H. Uchida, O. Aktsipetrov, A. Fedyanin, T. Murzina and A. Granovsky, Journal of Physics D: Applied Physics 39, R151 (2006).

[2] L. Wang, C. Clavero, Z. Huba, K. J. Carroll, E. E. Carpenter, D. Gu, R. A. Lukaszew, Nano Lett. 11, 1237 (2011).

[3] Y. Li, Q. Zhang, A. V. Numikko, and S. Sun, Nanolett. 5,1689 (2005).

[4] J. B. González-Díaz, A. García-Martín, G. Armelles, J. M. García-Martín, C. Clavero, A. Cebollada, R. A. Lukaszew, J. R. Skuza, D. P. Kumah, R. Clarke, Phys. Rev. B 76, 153402 (2007).

[5] H. Uchida, Y. Masuda, R. Fujikawa, A. V. Baryshev, M. Inoue, J. Magn. Magn. Mater. 321, 843 (2009).

[6] P. K. Jain, Y. Xiao, R. Walsworth, and A. E. Cohen, Nanolett. 9, 1644 (2009).

[7] K. M. Davis, K. Miura, N. Sugimoto, and K. Hirao, Opt. Lett. 21, 1729 (1996).

[8] Y. Shimotsuma, P.G. Kazansky, J. Qiu, K. Hirao, Phys. Rev. Lett. 91, 247405 (2003). 
[9] J. H. Kim, J. Kim, S. U. Lim, C. K. Kim, C. S. Yoon1, G. J. Lee, and Y. P. Lee, J. Appl. Phys. 99, 08G311 (2006).

[10]S. Nakashima, K. Fujita, A. Nakao, K. Tanaka, Y. Shimotsuma, K. Miura and K. Hirao, Appl. Phys. A 94, 83 (2009).

[11]S. Nakashima, K. Sugioka, and K. Midorikawa, Appl. Phys. A 104, 993 (2011).

[12] S. Nakashima, K. Sugioka, K. Tanaka, M. Shimizu, Y. Shimotsuma, K. Miura, K. Midorikawa, and K. Mukai, Opt. Express 20, 28191 (2012).
[13] S. Nakashima, K. Sugioka, K. Midorikawa, K. Mukai, Appl. Phys. A 110, 765 (2013).

[14]A. Bishay, J. Non-Cryst. Solids 3, 54 (1970).

[15] J. Qiu, X. Jiang, Q. Zhao, C. Zhu, H. Inoue, J. Si, and K. Hirao, Opt. Lett. 29, 370 (2004).

[16]K. Miura, J. Qiu, H. Inouye, T. Mitsuyu, K. Hirao, Appl. Phys. Lett. 71, 3329 (1997).

[17]X. Zhang, J. Schoenes, and P. Wachter, Solid. State Commun. 39, 189 (1981).

(Received: September 18, 2013, Accepted: April 14, 2014) 